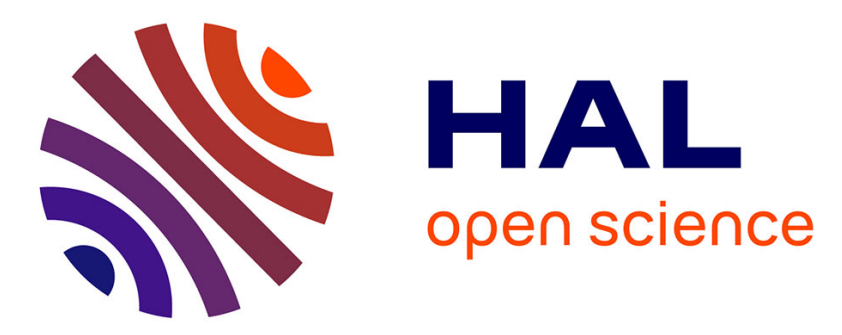

\title{
Kink-limited Orowan strengthening explains the brittle to ductile transition of irradiated and unirradiated bcc metals
}

\author{
T D Swinburne, S L Dudarev
}

\section{- To cite this version:}

T D Swinburne, S L Dudarev. Kink-limited Orowan strengthening explains the brittle to ductile transition of irradiated and unirradiated bcc metals. Physical Review Materials, 2018, 2 (7), 10.1103/physrevmaterials.2.073608 . hal-01961960

\author{
HAL Id: hal-01961960 \\ https://hal.science/hal-01961960
}

Submitted on 11 Feb 2019

HAL is a multi-disciplinary open access archive for the deposit and dissemination of scientific research documents, whether they are published or not. The documents may come from teaching and research institutions in France or abroad, or from public or private research centers.
L'archive ouverte pluridisciplinaire HAL, est destinée au dépôt et à la diffusion de documents scientifiques de niveau recherche, publiés ou non, émanant des établissements d'enseignement et de recherche français ou étrangers, des laboratoires publics ou privés. 


\title{
Kink-limited Orowan strengthening explains the brittle to ductile transition of irradiated and unirradiated bec metals
}

\author{
T. D. Swinburne* and S. L. Dudarev \\ UKAEA, CCFE, Culham Science Centre, Abingdon, Oxon OX14 3DB, United Kingdom
}

(Received 15 May 2018; published 23 July 2018)

\begin{abstract}
The line tension model of obstacle hardening is modified to account for the thermally activated, kink-limited glide of $1 / 2\langle 111\rangle$ screw dislocations, allowing application to the plastic flow of bcc metals. Using atomistically informed dislocation mobility laws, Frenkel-Kontorova simulations, and a simplified dislocation-obstacle model, we identify a size effect for intermediate obstacle densities, where the activation energy for screw dislocation motion halves once the obstacle density falls below a critical value. Our model shows striking agreement with fracture experiments across a wide range of unirradiated and irradiated bcc metals. In particular, we demonstrate that the presence of defects in the crystal lattice can at most double the brittle to ductile transition temperature.
\end{abstract}

DOI: 10.1103/PhysRevMaterials.2.073608

The motion of a dislocation line through a random field of static obstacles is a classic problem of theoretical metallurgy [1-7]. Whilst obstructions to dislocation motion in real materials are diverse, including forest dislocations, point defect clusters, solute atoms, precipitates, voids, and gas bubbles amongst others [8], many models do not focus on specifics of the dislocation-obstacle interaction mechanism [9-12], instead assigning a threshold obstacle bypass stress and then gauging the effect of the obstacle distribution on the flow stress [4]. The model is further simplified by treating the elastic self-energy of the dislocation, which is in principle given by a sum of an orientation-dependent line energy and its second derivative [13], by an isotropic line tension $\mu b^{2} / 2$, where $\mu$ is the shear modulus and $b$ is the Burgers vector of the dislocation [1]. The resultant model has a powerful generality which has been widely studied in materials science and statistical physics $[14,15]$.

The prototypical configuration of such models is shown in Fig. 1(a). An applied shear stress, $\sigma$, exerts a total force, $b \sigma L$, on a dislocation pinned between two obstacles spaced by $L$, resulting in the dislocation forming a circular arc with a radius of curvature $\mu b / 2 \sigma$. Obstacle bypass occurs once the total force on the obstacle pinning the dislocation line exceeds some defined threshold, $f_{\text {th }}$, allowing the dislocation to shear through the obstacle, or the total force causes the radius of curvature for two neighboring segments to fall below $L / 2$, whereupon the segments combine and "pinch off" $[1,8]$. The threshold condition $f_{\text {th }}=b \sigma_{\mathrm{F}} L$ thus yields $\sigma_{\mathrm{F}}=f_{\text {th }} /(b L)$ for a single segment. Applying this relation to an obstacle distribution, theoretical hardening laws and line tension simulations all predict that the flow stress $\sigma_{\mathrm{F}}$ obeys the famous Friedel relationship [3-7] $\sigma_{\mathrm{F}}=\alpha \mu b /\langle L\rangle$, where $\langle L\rangle$ is the average obstacle spacing and $\alpha$ is a dimensionless constant dependent on the obstacle distribution.

\footnotetext{
*tomswinburne@gmail.com; Current address: Theoretical Division T-1, Los Alamos National Laboratory, Los Alamos, NM 87545, USA.
}

Models of obstacle hardening are of clear relevance to irradiation-induced embrittlement, where under irradiation a relatively clean initial microstructure is populated with an increasing density of nanoscale defects, which impede dislocation motion [8]. The ability to predict the conditions under which irradiation induced embrittlement occurs remains a critical objective for nuclear materials science [16], in particular for body-centered-cubic (bcc) materials such as ferritic steels [17] and tungsten [18], where the brittle to ductile transition is known to be controlled by dislocation mobility [19,20]. Accurate modeling of obstacle hardening in bec metals is also essential to understand the ductility of oxide-dispersion-strengthened steels [21], which exhibit a large and unexplained brittleness and strength variability under current manufacturing techniques.

Plastic flow in bcc metals is famously controlled by the thermally activated, kink-limited, motion of $\langle 111\rangle$ screw dislocations [22-26]. Due to the large kink pair formation free energy $2 F_{k}(\sigma, T)$, the flow stress of bcc metals is highly temperature dependent even in the absence of obstacles, i.e., $\sigma_{\mathrm{F}} \rightarrow \sigma_{\mathrm{F}}^{0}(T)$ as $\langle L\rangle \rightarrow \infty$. The plasticity of bcc metals is thus controlled by an intrinsic lattice resistance even before the introduction of obstacles, complicating reconciliation with the phenomenology of line tension models, which assume that dislocation are free elastic lines before interaction with obstacles. In studies of bcc crystal plasticity [8,9,25-28], the Friedel stress $\alpha \mu b /\langle L\rangle$ is typically subtracted from the resolved shear stress to yield an effective shear stress which acts against the intrinsic lattice resistance. Using thermally activated dislocation mobility laws [8,24,26,27,29] the flow stress $\sigma_{F}$ instead emerges as that required to maintain a realistic strain rate.

In this paper, we revisit the classic line tension model of obstacle hardening, adding a lattice resistance to allow application to modeling bcc plasticity. The inclusion of a kink mechanism induces a qualitative change in dislocationobstacle interaction, as illustrated in Fig. 1(b). For realistic applied stresses (well below the Peierls stress [1]), dislocations do not bow out, as the lattice resistance counteracts the 
(a)

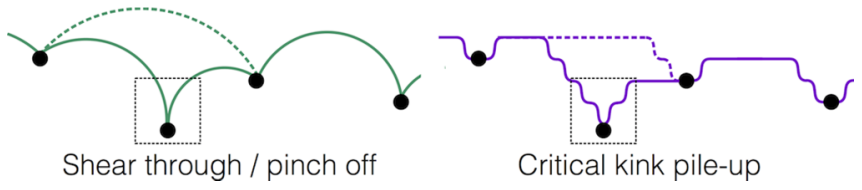

FIG. 1. (a) A simple elastic dislocation line pinned to obstacles under an applied stress. Obstacle bypass occurs once the applied stress exceeds a certain threshold value inversely proportional to the distance between obstacles. (b) The same model with a kink-mediated dislocation mobility mechanism. Obstacle bypass occurs once the stress on the kink pile-up exceeds a threshold that is independent of the obstacle separation, provided that the two pile-ups do not meet (see Fig. 2).

applied stress. Dislocation migration instead occurs through the thermal nucleation and propagation of kink pairs $[25,26]$, which form pile-ups at segment pinning points under an applied stress.

Previous studies of the influence of obstacles on kinklimited screw dislocation motion [8,30-32] have identified a size effect in the limit of densely distributed strong obstacles, where kink pile-ups forming on either side of pinned segments meet, as illustrated in Fig. 2(c). In this case, a segment of length $L$ can support up to $L /\left(2 w_{k}\right)$ kink pairs, where $w_{k}$ is the kink width. The total force exerted by the kink pile-ups forming on either side of a dislocation segment is $\sigma b h_{k} L / w_{k}$, where $h_{k}$ is the kink height. Equating the pile-up force to a defined threshold $f_{\text {th }}$, we find $\sigma=w_{k} f_{\text {th }} /\left(b h_{k} L\right)$, thus recovering the classical Friedel flow stress relationship $\sigma_{\mathrm{F}} \propto 1 /\langle L\rangle$. This has been demonstrated in dislocation dynamics simulations [30].

Our main result is the identification of a regime controlled by a well-known size effect in the kink nucleation rate $[1,27,33,34]$ operative on longer-length scales, where the activation energy for kink-limited dislocation motion halves from $2 F_{k}$ to $F_{k}$ once the average dislocation segment length $L$ becomes greater than a stress and temperature-dependent critical length:

$$
L^{*}(\sigma, T)=b \exp \left[\beta F_{k}(\sigma, T)\right] .
$$

(a)

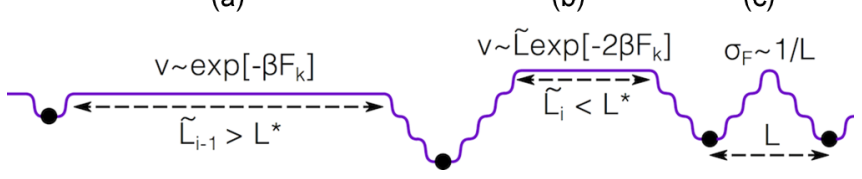

FIG. 2. Three regimes of kink-limited motion through obstacles. (a) The key regime identified in this work. When the available segment length $\tilde{L}>L^{*}$, Eq. (1), the screw dislocation velocity is length independent and has an activation energy equal to the formation energy of a single kink. (b) When $\tilde{L}<L^{*}$, the activation energy doubles and the velocity is linearly dependent on the segment length. In both cases, obstacle bypass occurs independently of the obstacle spacing. (c) When obstacles are sufficiently strong or closely spaced, the kink pile-ups meet before bypass and the dislocation cannot propagate. The critical stress in this regime [30] obeys the classical Friedel relationship $\sigma_{\mathrm{F}} \propto 1 / L$, where $L$ is the obstacle spacing.
In this regime, obstacle bypass still occurs once the total force $\sigma b h_{k} n_{k}$ exerted by a pile-up of $n_{k}$ kinks exceeds some defined threshold $f_{\text {th }}$, but the obstacle spacing is now sufficiently large, so that the obstacle bypass occurs before the opposing pile-ups meet, as shown in Fig. 2 (a). As a result, in the limit of dilute obstacles the flow behavior is almost independent of the obstacle spacing. For higher obstacle densities the effective dislocation length for kink nucleation is restricted, causing a doubling of the kink nucleation energy and a lengthdependent dislocation velocity, restricting the rate of plastic flow, as shown in Fig. 2(b). Through multiscale simulations and comparison to a wide range of experimental data, we show that this provides a powerful model for understanding irradiation-induced embrittlement.

Using the Frenkel-Kontorova model [33] and kink-limited dislocation-obstacle simulations, we evidence a modified Orowan flow law $[25,27,35]$ :

$$
\dot{\epsilon}= \begin{cases}\rho b\langle L\rangle \omega_{0} \exp \left[-2 \beta F_{k}(\sigma, T)\right] & \langle L\rangle \leqslant L^{*}(\sigma, T), \\ \rho b^{2} \omega_{0} \exp \left[-\beta F_{k}(\sigma, T)\right] & \langle L\rangle \geqslant L^{*}(\sigma, T),\end{cases}
$$

where $\rho$ is the dislocation density. Employing dislocation mobility laws for unirradiated materials parametrized from atomistic simulations [25,27,36], we show that under temperatures and applied stresses appropriate for the brittle to ductile transition (BDT), the critical length $L^{*}$ falls to values of order $10^{-1} \mu \mathrm{m}$, well within the range of typical obstacle spacings $\langle L\rangle$. As illustrated in Fig. 2, the effective activation energy for dislocation motion thus doubles once the density of obstacles increases and the characteristic obstacle spacing $\langle L\rangle$ falls below $L^{*}$, a phenomenon which signifies the onset of embrittlement.

We find compelling agreement with our model through comparison with experimental measurements of the brittle to ductile transition temperature (BDTT) in a wide range of high-purity bcc metals [37] and neutron-irradiated lowactivation steels [17]. In particular, the single-kink-activation energy flow law is in very good agreement with experimental data on pure materials. This signature is entirely compatible with low-temperature bcc plasticity studies [28], which find a double-kink activation energy as a characteristic parameter in the dislocation mobility law, as in the low-temperature regime explored in Ref. [28] the critical length $L^{*}$ is very large, of order $10^{3}-10^{4} \mu \mathrm{m}$, and thus the activation-energy-related BDT transition remains outside the range of parameter space spanned by observations. Applying our model to irradiated materials we find that the BDTT can at most double due to the presence of obstacles, a powerful qualitative relationship which is clearly obeyed in fracture experiments on neutronirradiated low-activation steels of widely varying composition.

The paper is structured as follows. In Sec. I we explore Frenkel-Kontorova simulations of pinned screw dislocation segments, where the transition in the activation energy for dislocation motion as a function of dislocation segment size is confirmed. In Sec. II we review dislocation mobility laws for unirradiated materials, parametrized from atomistic simulations $[25,27,36]$ and produce quantitative estimates for the stress- and temperature-dependent critical length $L^{*}(\sigma, T)$. In Sec. III we condense the observed phenomenology into a simplified dislocation-obstacle interaction model to obtain robust statistical data on the transition in screw dislocation 
velocity. Finally, in Sec. IV we compare the predictions of our model to temperature-controlled fracture experiments performed on a wide range of unirradiated and irradiated bcc metals.

\section{FRENKEL-KONTOROVA SIMULATIONS OF PINNED SCREW DISLOCATIONS}

The phenomenology of kink-limited dislocation motion has been extensively studied $[28,36,38]$ using the stress-driven Frenkel-Kontorova (FK) model [33], which for a line of $N$ nodes with slip plane positions $\left(x_{n}, y_{n}\right)=\left(b n, h \xi_{n}\right)$ has the potential energy

$$
E=\sum_{n} \frac{\kappa h^{2}}{2 b^{2}}\left(\xi_{n+1}-\xi_{n}\right)^{2}+V \sin ^{2}\left(\pi \xi_{n}\right)-b^{2} h \sigma \xi_{n},
$$

where $\kappa$ is the line energy of the FK model, $V$ the Peierls barrier, and $\sigma$ the applied stress. Without loss of generality, we choose energy units of $V$ and length units of $b$. With $h=b$ this system has a Peierls stress of $\sigma_{p}=\pi V / b^{3}$; setting $\kappa=200 \mathrm{~V}$ gives a kink energy of $U_{k}=40 \mathrm{~V} / \pi$ and a kink width parameter of $10 \mathrm{~b} / \pi$, yielding the highly mobile kinks appropriate for $\langle 111\rangle$ screw dislocations in bec metals [36]. For the Frenkel-Kontorova model, the kink free energy is given by $F_{k}(\sigma, T) \sim U_{k}\left(1-\sigma / \sigma_{p}\right)$ for small stresses [24]. In the kink-limited regime, the line velocity $v$ is simply related to the net kink nucleation rate in the direction of the applied stress $\bar{\Gamma}=\Gamma(\sigma)-\Gamma(-\sigma)$ through $v=b \bar{\Gamma}[25]$. Dynamics are generated using the overdamped Langevin equation with either periodic boundary conditions $\xi_{N}=\xi_{0}$ or pinned boundary conditions $\xi_{N}=\xi_{0}=0$, taking ensemble averages to produce robust statistical data [39].

A typical individual simulation configuration is illustrated in Fig. 3(a). For migration distances up to around $25 \%$ of the line length, we find the velocity of a pinned line is comparable to that of a periodically repeated dislocation segment of the same length, confirming that the only effect of the pinned boundary conditions is to create two kink pile-ups, with no effect on the bulk of the line. Figure 3(b) demonstrates that for short lines $\bar{\Gamma} \propto L$, but above a certain length threshold $L^{*}(\sigma, T)$, it becomes length independent. By comparing similar simulations at a range of temperatures, one can extract an activation energy from the slope of an Arrhenius plot $(\beta, \ln \bar{\Gamma})$ [40]. Combining these simulations, we confirm that for short segments $\Gamma(\sigma) \sim(L / b) \exp \left[-2 \beta F_{k}(\sigma)\right]$, whilst for longer segments $\Gamma(\sigma) \sim \exp \left[-\beta F_{k}(\sigma)\right]$, the activation energy thus halving for longer lines [Fig. 3(c)]. It is also possible to observe the crossover at a fixed line length simply by varying the temperature and applied stress, as shown in Fig. 3(d). In agreement with literature data $[1,27,34]$, we find that the crossover length $L^{*}(\sigma, T)$ is well approximated by Eq. (1), $L^{*}(\sigma, T)=b \exp \left[\beta F_{k}(\sigma, T)\right]$. We provide a derivation of the crossover length in Appendix A.

The dislocation segment size effect on the kink nucleation rate found in our simulations is well known [1,27,33,34]. Here, the dependence of the kink nucleation rate $\bar{\Gamma}$ on the segment length $L$ forms the central hardening mechanism in our model, with the crossover length (1) being the critical length scale
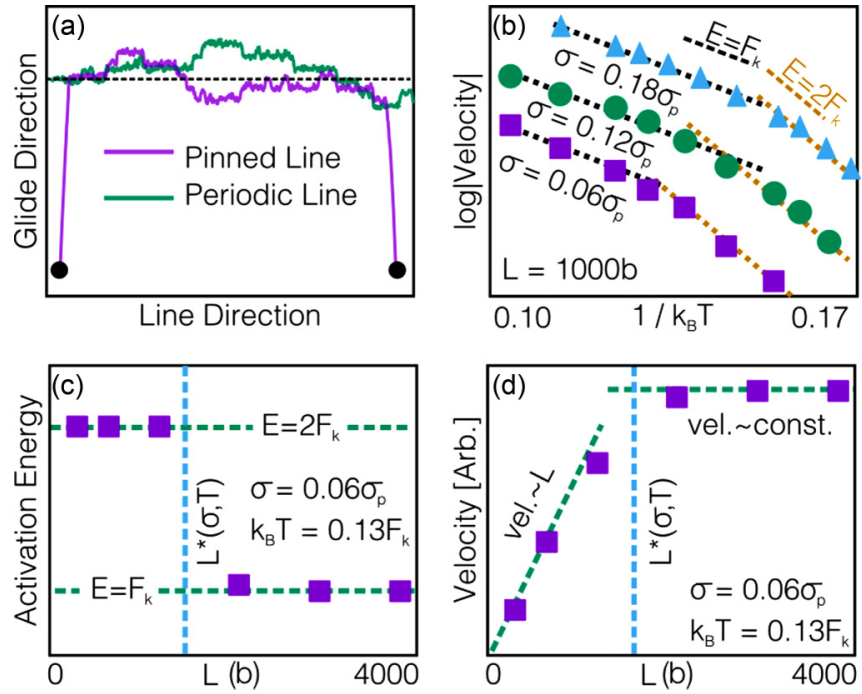

FIG. 3. Frenkel-Kontorova model simulations showing size effects in the kink nucleation rate. (a) A pinned and unpinned FrenkelKontorova dislocation under applied stress. (b) Arrhenius plot of the average velocity. The activation energy halves at high temperature. (c) The activation energy halves for segments longer than the critical length $L^{*}(\sigma, T)$. (d) The velocity is proportional to the segment length $L$ below $L^{*}(\sigma, T)$ and is independent of length above $L^{*}(\sigma, T)$.

when comparing to experiment, to a large degree independent of the details of dislocation-obstacle interaction.

\section{KINK-FORMATION FREE ENERGY}

Whilst our Frenkel-Kontorova simulations can capture the statistics of the kink nucleation rate as a function of segment length and kink-formation energy over a wide parameter range, the intrinsic simplicity of the model clearly cannot reproduce the complex stress and temperature dependence found in fully atomistic simulations [25,41-43]. In particular, the kink-formation free energy and flow stress are known to vanish at both the so-called "athermal" temperature $T_{\text {ath }}$ $[24,29]$ and a temperature-dependent flow stress $[8,24,26,27]$. Accommodation of these features is known to be essential to accurately model high-temperature screw dislocation motion and thus capture experimental data. Recent calculations of the zero-stress double-kink-formation free energy in tungsten confirms the large contribution of vibrational entropic terms, which are not predicted in harmonic approximations using static curvatures [43]. Following previous studies, a general approximate form of the kink-formation free energy reads $[8,24,26,27,29]$

$$
F_{k}(\sigma, T)=U_{k}\left(1-\frac{T}{T_{\text {ath }}}-\frac{\sigma / \sigma_{p}}{1-T / T_{\text {ath }}}\right) .
$$

In Fig. 4(a) we plot Eq. (4) for values appropriate for bcc iron. It is clear that for realistic applied stresses $(\sigma \lesssim 200 \mathrm{MPa})$, an Arrhenius plot of $\left(-F_{k} / k_{\mathrm{B}} T, 1 / k_{\mathrm{B}} T\right)$ yields a slope that varies by less than $10 \%$ from the zero-stress, zero-temperature kinkformation energy $U_{k}$ for realistic resolved shear stresses (150 MPa) present under typical experimental strain rates $\dot{\epsilon} \lesssim 10^{-4}$ $\mathrm{s}^{-1}$, demonstrating that the stress and temperature dependence of Eq. (4) predominantly affects the entropy (i.e., prefactor) of 

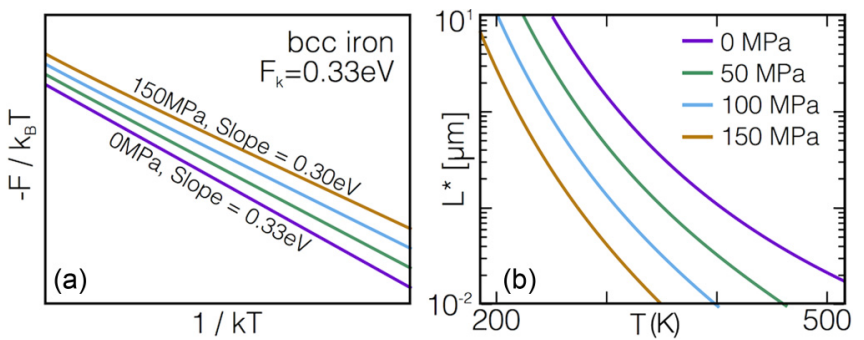

FIG. 4. (a) Arrhenius plot $\left(-F_{k} / k_{\mathrm{B}} T, 1 / k_{\mathrm{B}} T\right)$. (b) Crossover length $L^{*}$ (1), computed using the approximate kink-formation free energy (4), with values of $U_{k}=0.33 \mathrm{eV}, \sigma_{p}=900 \mathrm{MPa}$, and $T_{\text {ath }}=700 \mathrm{~K}$ appropriate for a screw dislocation in Fe [29]. Applied stress and temperature have dramatic effects on $L^{*}$, but the effective activation energy changes by less than $10 \%$.

kink nucleation. The consequences of this entropic boost can be clearly seen, however, when using Eq. (4) in our expression (1) for the crossover length $L^{*}$, being the characteristic length scale where the effective activation energy for plastic flow halves. As illustrated in Fig. 4(b) for bcc iron, under realistic stresses and temperatures the crossover length can be as low as $10^{-2}$ $\mu \mathrm{m}$, whilst pure materials typically have dislocation mean free paths of the order of micrometers [8]. The fact that the crossover length $L^{*}$ is comparable to typical microstructural length scales is a key factor in the stress, temperature, and obstacle density dependence of the brittle to ductile transition and is a key conclusion of this paper.

\section{DISLOCATION-OBSTACLE MODEL}

To probe the effect of a random obstacle array on screw dislocation mobility we have used a simplified dislocationobstacle model which captures the phenomenology of our Frenkel-Kontorova simulations. An initially straight dislocation propagates into an array of obstacles, where it is pinned and split into multiple segments of lengths $\left\{L_{i}\right\}$. As has been noted in previous studies [30-32], when a dislocation segment propagates a distance $d$, the finite kink width $w$ reduces the effective segment length for further kink nucleation to $\tilde{L}_{i}=L_{i}-2 w d / h$, meaning that no segment can propagate further than $h L_{i} / 2 w$, as illustrated in Fig. 2. Using the results above, we assign to each segment a velocity

$$
v(\tilde{L}, \sigma, T)=\omega_{0} b^{2}\left[\frac{1}{L^{*}(\sigma)}-\frac{1}{L^{*}(-\sigma)}\right] \frac{\min \left(L^{*}, L\right)}{L^{*}(\sigma)},
$$

where $F_{k}(\sigma, T)$ is taken from Eq. (4). Without loss of generality, we set $\sigma>0$. In the rare event limit, the $1 / L^{*}(-\sigma)$ term has a negligible contribution to Eq. (5), upon which $\rho b v(\langle L\rangle, \sigma, T)$ is precisely the Orowan flow law (2).

In each realization of our simulations, a simulation cell of width $W$ and height $H$ is populated with a uniformly random array of $N$ obstacles with an initially straight dislocation line lying at the bottom of the cell. The dislocation line is propagated forward a distance $d_{0}$ until an obstacle is met, and then the global clock time is updated to $t_{\mathrm{G}}=d_{0} / v(W, \sigma, T)$ and the dislocation line is divided into segments.

A pinned segment of length $L$ will propagate a distance $v\left(\tilde{L}_{i}\right) \Delta t$ in a time $\Delta t$, creating an additional $v\left(\tilde{L}_{i}\right) \Delta t / h_{k}$ kink

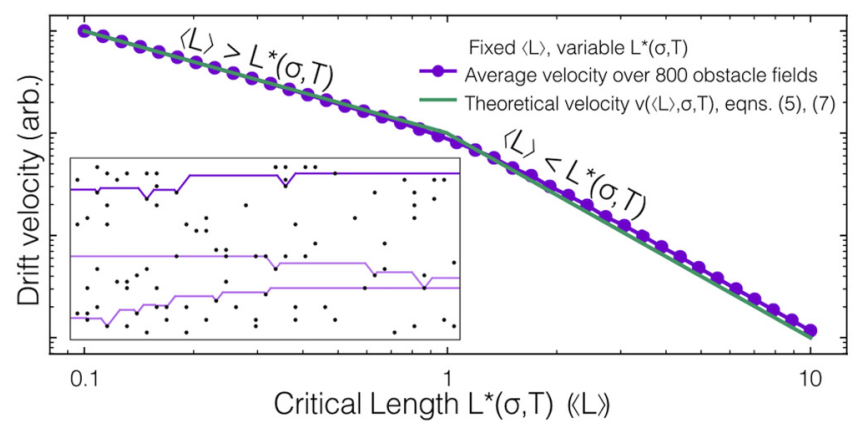

FIG. 5. Predicted and calculated ensemble average velocity with varying critical length at fixed obstacle density, equivalent to varying density at a fixed critical length. Inset: Sample configurations from coarse-grained model of screw dislocation-obstacle interactions. Kink pile-ups are approximated by angled lines rather than steps for computational efficiency.

pairs, which pile up at the segment ends. As discussed above, the total force exerted by a kink pile-up of height $n h_{k}$ on an obstacle is given by $n \sigma b h_{k}$. With the threshold obstacle force $f_{\text {th }}$, the segment will bypass the obstacle once the number of kinks in the pile-up exceeds

$$
n_{\mathrm{th}}=\frac{f_{\mathrm{th}}}{\sigma b h_{k}} .
$$

In practice, there will be two kink pile-ups of size $n_{i}^{\mathrm{L}}\left(n_{i}^{\mathrm{R}}\right)$ on each side of a dislocation segment. When any pile-up exceeds $n_{\text {th }}$, the two segments on either side of the obstacle are combined, with the remaining kinks propagated to the ends of the new joined segment. The maximum number of kink pairs that the segment can support [as illustrated in Fig. 2(c)] is given by $L_{i} /\left(2 w_{k}\right)$. If the corresponding pile-up height $L_{i} h_{k} /\left(2 w_{k}\right)$ is less than $n_{\text {th }}$, the segment will remain pinned until a neighboring segment breaks free. If all the segments are pinned in this manner, the applied stress is not sufficient to induce plastic flow; i.e., we are below the Friedel flow stress, which we ensured did not occur in the simulations used here.

The simulation algorithm is therefore as follows. For each segment $i$ of available nucleation length $\tilde{L}_{i}$, the distance $\tilde{d}_{i}$ to the nearest obstacle is calculated, giving a segment collision time of $\tilde{t}_{i}=\tilde{d}_{i} / v\left(\tilde{L}_{i}\right)$. The expression for the collision time, accounting for the length-dependent mobility, is given in Appendix B. The smallest collision time $t_{\min }=\min \left\{\tilde{t}_{i}\right\}$ updates the global clock time $t_{\mathrm{G}} \rightarrow t_{\mathrm{G}}+t_{\mathrm{min}}$; each segment migrates a distance $v\left(\tilde{L}_{i}\right) t_{\mathrm{min}}$, with one segment thus bisected by an obstacle. If the total migration distance past the obstacle on the left or right of a segment is greater than $n_{\mathrm{th}} h_{k}$, then the neighboring segments are combined and the remaining kinks move to the segment ends. Typical simulation snapshots are shown in the inset of Fig. 5.

Using this simulation procedure, we can extract the average dislocation velocity and effective activation energy, characterizing plastic flow across a wide range of applied stresses, temperatures, obstacle densities, and obstacle strengths. The results of these simulations are shown in Fig. 5. For low obstacle densities, such that the obstacle spacing is greater than $L^{*}$, the dislocation velocity is initially unchanged from the obstacle-free lattice case, with an activation energy being 
equal to the single-kink-formation energy. However, above a threshold obstacle density (see below), the dislocation velocity drops rapidly and the characteristic activation energy doubles.

To recover the same dislocation velocity, the temperature of the system has to effectively double. In the next section we see that all of this phenomenology is exhibited in fracture experiments on unirradiated and irradiated bcc metals.

To predict the dependence of dislocation motion on the density of obstacles, we require an analytical expression for the expected pinned segment length $\langle L\rangle$. Dislocation glide is modeled as planar in a given slip system in our simulations, meaning that a random point obstacle distribution in three-dimensional space with an average density $\rho_{\text {obs }}$ has an average density of $c=\rho_{\mathrm{obs}} s$ on the glide plane, where $s$ is the characteristic obstacle size, typically from $4 b$ to $10 b$. As a dislocation segment will depin from an obstacle once the kink pile-up height exceeds $n_{\text {th }} h_{k}$, we thus ask for the length $\langle L\rangle$ of a pinned dislocation with $n_{t h}$ kinks whose swept area contains a single obstacle. In Appendix $\mathrm{C}$ we show this is given by

$$
\frac{\langle L\rangle}{w_{k}}=\frac{n_{\mathrm{th}}}{2}\left[\frac{\operatorname{erf}(\sqrt{\phi})}{2 \sqrt{\phi / \pi}}+\frac{2+\phi}{\phi} e^{-\phi}\right], \phi=c n_{\mathrm{th}}^{2} w_{k} h_{k},
$$

where $\operatorname{erf}(x) \equiv(2 / \sqrt{\pi}) \int_{0}^{x} \exp \left(-y^{2}\right) d y$ is the error function. This expression takes simple forms in the limits where the kink width $w_{k}(\sim \sqrt{\kappa / V}$ for a line tension $\kappa$ and Peierls barrier $V$ [36]) is much larger or smaller than the obstacle spacing. In the limit of a large kink width, the swept area is approximately a triangle, yielding $\langle L\rangle=\sqrt{\pi n_{\mathrm{th}} w_{k}} /\left(4 \sqrt{c h_{k}}\right)$, whilst for a small kink width the swept area is approximately a rectangle, yielding $\langle L\rangle=1 /\left(c n_{\mathrm{th}} h_{k}\right)$.

In Fig. 5, we plot the results of simulations using a fixed obstacle density of $\rho_{\text {obs }} s \simeq 1 /\left(1500 b w_{k}\right)$, and thus a fixed $\langle L\rangle$, capturing the effect of variable temperature and stress by varying $L^{*}$ by around an order of magnitude above and below $\langle L\rangle$. Whilst this phenomenological model has no inherent length scale, using values appropriate for bcc iron shown in Fig. 4(b), we see that the simulation supercell has the dimensions of $10-1000 \mu \mathrm{m}$.

For each value of $L^{*}$ the average drift velocity was obtained across the same set of 800 independently generated obstacle distributions, uniformly distributed across a discrete grid to avoid very close obstacles (simulating athermal annealing in a real material $[44,45])$. As can be seen, the theoretical prediction (5) using Eq. (7) for $\langle L\rangle$ gives excellent agreement with our simulations, showing that the length-dependent mobility of individual pinned dislocation segments clearly transfers to directly determine the average velocity of dislocation lines moving with kink-limited mobility through a field of obstacles. In particular, for low obstacle densities, where $\langle L\rangle<L^{*}$, we see that the dislocation velocity is unaffected by the obstacle field, giving a clear single-kink activation energy. This behavior is confirmed in the next section, where we compare our model to experimental data.

\section{COMPARISON TO EXPERIMENTS ON PURE AND IRRADIATED BCC METALS}

In this section we compare predictions derived from our model to strain- and temperature-dependent measurements of the BDT temperature $T_{\mathrm{BDT}}$ in a wide range of high-purity single-crystal bcc metals [37] and irradiated low-activation ferritic-martensitic steels of great practical importance to the fission and fusion applications [17]. In the experiments, small bars of the candidate material are subject to either bending [37] or impact [17] tests at a controlled temperature until the sample fractures; in the bending tests the strain rate can also be controlled. The degree of ductility in impact tests is determined by the amount of absorbed energy before fracture. It is well known [19] that in the bending experiments the character of failure mode undergoes a step change as a function of temperature, varying from brittle cleavage at low temperature to semibrittle fracture to ductile bending over a narrow temperature range termed the brittle to ductile transition, with the center of the transition region giving the $\mathrm{BDT}$ temperature $T_{\mathrm{BDT}}$.

As embrittlement is known to be controlled by dislocation mobility in bcc metals $[19,20]$, the strain rate $\dot{\epsilon}$ and the temperature $T_{\mathrm{BDT}}$ at which ductile fracture occurs are often interpreted using the Orowan flow law $[25,27,35] \dot{\epsilon}=\rho b^{2} v$. This is equivalent to the statement that ductile fracture occurs at the brittle to ductile transition temperature $T_{\mathrm{BDT}}$ once dislocation motion can relax the externally applied stresses sufficiently rapidly to avoid failure. Using expression (2) for the Orowan flow law appropriate for unirradiated materials, where the dislocation mean free path is large and $\langle L\rangle>L^{*}$, our model predicts the simple Arrhenius relation for the unirradiated brittle to ductile transition:

$$
\log \left|\dot{\epsilon}_{\text {unirr }}\left(T_{\mathrm{BDT}}\right)\right|=-\frac{U_{k}}{T_{\mathrm{BDT}}}+S_{k}+\ln \left|\rho b^{2} \omega_{0}\right| .
$$

Equation (8) is a main result of this paper, predicting that the characteristic activation energy for ductile fracture in the low obstacle density materials is the single-kink-formation energy $U_{k}=F_{k}+T S_{k}$. As discussed above and illustrated in Fig. 4, the large kink-formation entropy $S_{k}$ significantly reduces the crossover length $L^{*}$ to submicron values, i.e., well within the $\langle L\rangle>L^{*}$ criterion, but due to a fairly weak temperature dependence we find that the observed activation energy should still be the single-kink-formation energy $U_{k}$.

We find remarkable agreement with the prediction of Eq. (8) across a range of high-purity unirradiated bcc metals. Figure 6 plots the experimentally determined activation energy for ductile fracture against the calculated single-kink-formation energy using atomistic simulations [36,38], showing extremely tight correlation. The similar fracture behavior for single and polycrystalline tungsten and iron confirms that the dominant fracture mode is transgranular and thus controlled by dislocation mobility. We emphasize that the clear evidence for a single-kink-formation energy strongly supports a fundamental underlying mechanism of our model, that the characteristic activation energy for kink nucleation halves for long screw dislocation segments.

Having verified the predictions of our model on the BDT of unirradiated bcc metals, we now apply it to the interpretation of experiments studying irradiation-induced shifts in the brittle to ductile transition temperature $T_{\mathrm{BDT}}$ in low-activation steels. Due to the greater range of factors involved in the sample preparation, experimental data sets for irradiated materials invariably consider an output parameter space smaller than that of those for unirradiated materials, complicating detailed interpretation. In particular, changes in the BDTT are typically 


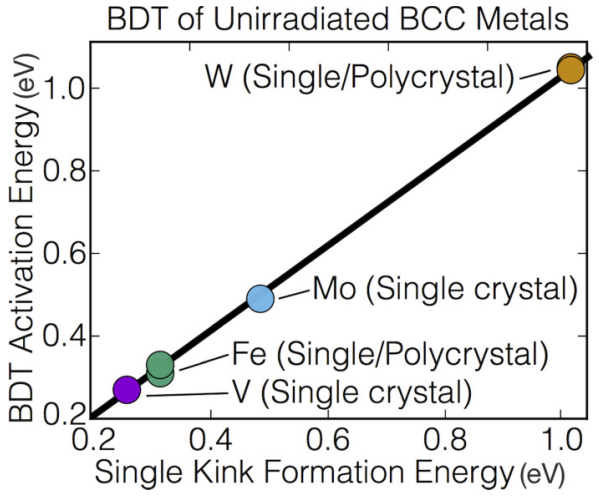

FIG. 6. Plot showing the agreement between characteristic activation energies for the BDT temperature $T_{\mathrm{BDT}}$ of high-purity bcc metals, derived from an Arrhenius plot of $T_{\mathrm{BDT}}$ versus strain rate [37], with the single-kink-formation energy predicted by atomistic simulations $[36,38]$. This relationship is predicted by Eq. (8) of our model, strongly supporting our conclusion that the characteristic activation energy for kink nucleation halves for long screw dislocation segments.

determined through Charpy impact tests, where the absorbed energy is measured as a function of sample temperature, meaning the imposed strain rate cannot be resolved.

In the experimental data considered here [17], samples of low-activation ferritic-martensitic steels were subjected to neutron irradiation to a fixed dose under a fixed irradiation temperature $T_{\text {irr }}$ before Charpy impact tests were performed at a range of temperatures. The absorbed energy measured in a Charpy impact test is well known to sharply increase with increasing sample temperature, which is associated with a ductile response [19]; fitting a smooth step function to these data then determines the BDTT. The fracture was observed to be transgranular, confirming that dislocation mobility, as opposed to grain boundary strength, controlled the mechanical response. As defect recombination and annealing rates increase with $T_{\text {irr }}$ [46], positive shifts in the BDTT associated with the accumulation of radiation defect clusters should be suppressed by increasing $T_{\text {irr }}$ as the obstacle density decreases, and the characteristic scale of the microstructure becomes larger due to defect cluster coarsening.

Figure 7 shows the resultant BDTT data from these experiments [17]. A dramatic manifestation of the qualitative behavior described above is clearly observed. The BDTT (expressed in Kelvin units) approximately doubles if the steel samples are exposed to irradiation at relatively low temperature, generating a high density of irradiation defect clusters impeding the motion of dislocations, but sharply returns to approximately the unirradiated value over a narrow window of around $50 \mathrm{~K}$ in $T_{\text {irr }}$.

Importantly, this behavior is seen across a range of steels with a variety of impurity compositions, indicating that the underlying physics of a sharp doubling of $T_{\mathrm{BDT}}$ with irradiation dose if steels are exposed to irradiation at low temperatures is an intrinsic fundamental property of the crystal microstructure rather than the specifics of a particular impurity-defect interaction.

The observed behavior can be simply understood in our model. It is clear that a ductile response to the Charpy impact

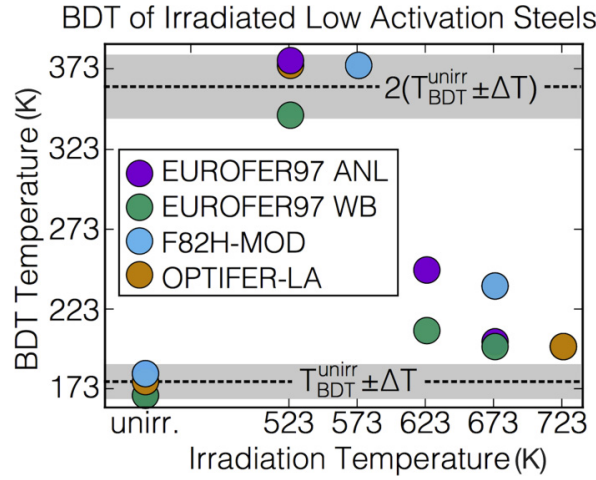

FIG. 7. Observed values of $T_{\mathrm{BDT}}$ in various irradiated reducedactivation ferritic-martensitic steels [17], which contain a diverse range of alloying elements. In agreement with the inequality relation predicted by our model, Eq. (9), $T_{\mathrm{BDT}}$ at most doubles under high obstacle density (low-irradiation temperature), but then returns back to the value characteristic of unirradiated steel as the obstacle density decreases (high-irradiation temperature). Importantly, this behavior is largely independent of the presence of alloying elements.

test requires similar dislocation velocities for irradiated and unirradiated materials, meaning we can equate the strain rate at the BDTT. In the unirradiated case, the temperature required for ductile flow is $T_{\mathrm{BDT}}^{\mathrm{unirr}} \simeq 173 \mathrm{~K}$. Using the modified Orowan law (2) validated in our simulations, the temperature of the brittle to ductile transition in an irradiated material $T_{\mathrm{BDT}}^{\mathrm{irr}}$ can be expressed as

$$
T_{\mathrm{BDT}}^{\mathrm{irr}}=\frac{2 F_{k}}{F_{k} / T_{\mathrm{BDT}}^{\mathrm{unirr}}+\ln \left|\langle L\rangle / w_{k}\right|} \leqslant 2 T_{\mathrm{BDT}}^{\mathrm{unirr}},
$$

where the upper bound corresponds to the limit of high obstacle density and thus small values of $\langle L\rangle$. Equation (9) is a central result of this paper, an upper bound for irradiation induced shifts in the brittle to ductile transition temperature, determined largely by geometric properties of the obstacle distribution.

Using values appropriate for bcc iron and thus ferretic steels, we find that $T_{\mathrm{BDT}}^{\mathrm{irr}}>1.8 T_{\mathrm{BDT}}^{\mathrm{unirr}}$ for $\langle L\rangle<100 \mathrm{~b}$, showing that the upper limit is valid for a wide range of obstacle densities. This provides a clear rationalization of the available experimental data, which also shows that the $T_{\mathrm{BDT}}^{\mathrm{irr}}$ is bounded from above by $2 T_{\mathrm{BDT}}^{\mathrm{unir}}$. We also note that the return of the BDT temperature to its value characteristic of unirradiated steels in the limit where samples were exposed to irradiation at high temperature does not imply the absence of obstacles; all that is required for the recovery of the BDT temperature to its original low value is the decrease of the volume density of obstacles, resulting, for example, from coarsening of the microstructure.

\section{CONCLUSIONS}

In this paper we have introduced a theory of obstacle hardening for bcc metals, which accounts for the thermally activated flow of $1 / 2\langle 111\rangle$ screw dislocations. Via multiscale simulation and theoretical analysis of thermally activated plastic flow through a random obstacle array, we show that the characteristic activation energy for plastic flow halves when the average obstacle spacing is above a well-defined threshold value, which depends only weakly on the specifics of 
dislocation-obstacle interaction and is dominated by the kink formation energy.

Our model predicts that the characteristic activation energy for ductile fracture is the single-kink-formation energy, in excellent agreement with fracture experiments across multiple bcc metals. We also predict that the brittle to ductile transition temperature (BDTT) increase following irradiation amounts to at most doubling the BDTT of an unirradiated material, which is also in agreement with fracture experiments on low-activation ferritic-martensitic steels. The powerful relationships revealed by our analysis should aid the design of radiation-resistant materials; the use of the above model to give quantitative predictions of irradiation-induced shifts in the BDTT, or quantitative predictions of hardening of oxidedispersion-strengthened steels, requires separate estimates of characteristic obstacle distribution, which will be the topic of future work.

\section{ACKNOWLEDGMENTS}

The authors thank both anonymous referees for helpful comments on an earlier draft of this paper. This work has been carried out within the framework of the EUROfusion Consortium and has received funding from the Euratom Research and Training Programme 2014-2018 under Grants No. 633053 and No. 755039. Also, it has been partially funded by the RCUK Energy Programme (Grant No. EP/P012450/1). The views and opinions expressed herein do not necessarily reflect those of the European Commission. S.L.D. is grateful to I. Lindsey and M. M. E. Coolsen for their critically significant contribution that enabled this research to be carried out.

\section{APPENDIX A: DERIVATION OF THE CROSSOVER LENGTH}

In steady-state dislocation glide, where kink nucleation is a rare event, the kink population is in thermal equilibrium and the drift velocity is proportional to the number of kinks [1]. As kinks can only be produced in pairs, the kink contribution to the partition function for a line of length $L=\tilde{N} b$ reads

$$
\begin{aligned}
Z & =\sum_{r=0}^{\infty} \frac{\tilde{N} ! \exp \left(-2 r \beta F_{k}\right)}{(\tilde{N}-r) ! r !} \\
& =\frac{1}{2}\left[1+\exp \left(-\beta F_{k}\right)\right]^{\tilde{N}}+\frac{1}{2}\left[1-\exp \left(-\beta F_{k}\right)\right]^{\tilde{N}},
\end{aligned}
$$

where the last equality uses the binomial expansion. The expected number of kinks in thermal equilibrium is given by

$$
\begin{aligned}
\left\langle n_{k}\right\rangle & =-\beta^{-1} \frac{\partial}{\partial F_{k}} \log Z \\
& =\tilde{N} \frac{e^{-\beta F_{k}}\left(1+e^{-\beta F_{k}}\right)^{\tilde{N}-1}-e^{-\beta F_{k}}\left(1-e^{-\beta F_{k}}\right)^{\tilde{N}-1}}{\left(1+e^{-\beta F_{k}}\right)^{\tilde{N}}+\left(1-e^{-\beta F_{k}}\right)^{\tilde{N}}},
\end{aligned}
$$

which has two limiting cases of interest, namely the thermodynamic limit $\tilde{N} \rightarrow \infty$ and the low-temperature limit $\beta \rightarrow$ $\infty$. As both appear as powers (A2), these limits will be competing; the thermodynamic limit will be harder to reach at low temperature and vice versa. In the thermodynamic limit $\tilde{N} \rightarrow \infty$ we find that

$$
\lim _{\tilde{N} \rightarrow \infty} \frac{\left(1-e^{-\beta F_{k}}\right)^{\tilde{N}}}{\left(1+e^{-\beta F_{k}}\right)^{\tilde{N}}}=0,
$$

giving an expected kink population of

$$
\left\langle n_{k}\right\rangle \rightarrow \frac{\tilde{N}}{\exp \left(\beta F_{k}\right)+1} \sim \tilde{N} \exp \left(-\beta F_{k}\right),
$$

which is the expected Fermi distribution for single kinks. However, in the rare event limit of interest in this work, the Boltzmann factor $\exp \left(-\beta F_{k}\right)$ is small $\left(\sim 10^{-6}\right.$ for room temperature $\mathrm{Fe})$. To the fourth order in the Boltzmann factor we can make the expansion

$$
\left\langle n_{k}\right\rangle \rightarrow \tilde{N}(\tilde{N}-1) \exp \left(-2 \beta F_{k}\right),
$$

which has the double-kink energy. To see where the thermodynamic limit is expected to compete with the low-temperature limit, we look for the system size for which $\left\langle n_{k}\right\rangle \sim 1-2$ and solve for $\tilde{N}$. Approximating $\tilde{N}(\tilde{N}-1) \simeq \tilde{N}^{2}$ we find an approximate crossover length $L^{*}$ given by

$$
L^{*} \sim b \exp \left(\beta F_{k}\right)
$$

Whilst the precise value of the multiplicative factor in front of the exponential will depend on the definition of the crossover kink population, the key feature is that the crossover kink population will be independent of the kink-formation energy, yielding a crossover length that scales as $\exp \left(\beta F_{k}\right)$, which is central to the phenomenon that we study.

\section{APPENDIX B: DERIVATION OF THE SEGMENT COLLISION TIME}

Consider a dislocation segment with an available kink nucleation length $\tilde{L}_{i}$, as illustrated in Fig. 8. Under an applied stress the line will nucleate and accumulate kinks, eventually (in the absence of obstacles or detachment) forming a triangle of height $\tilde{L}_{i} / \alpha$, where $\alpha=w_{k} / h_{k}$ is the ratio of kink width to kink height. A finite collision time is therefore only possible if an obstacle lies inside this triangle; we consider such a case, with the obstacle lying a glide distance $d$ away from the line segment. Using the rare event limit of the velocity law (5) and defining $v_{0} \equiv \omega_{0} b \exp \left[-\beta F_{k}(\sigma)\right]$ we have three cases for the

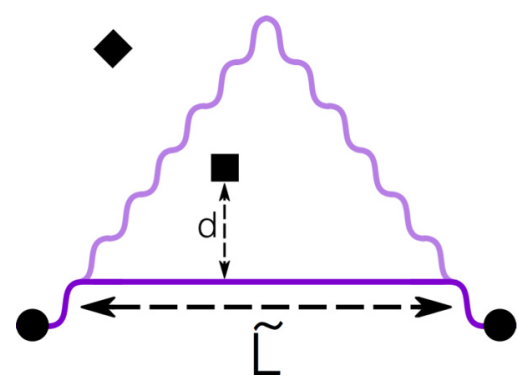

FIG. 8. Illustration of segment collision time calculation. The maximum propagation state is shown as a transparent line. The diamond obstacle has an infinite collision time as it lies outside the propagation state. 
collision time:

$$
\Delta t(d)= \begin{cases}-\frac{L^{*}}{2 \alpha v_{0}} \log \left[1-\frac{2 \alpha d}{L^{*}}\right], & \tilde{L} \leqslant L^{*}, \\ \frac{\tilde{L}-L^{*}}{2 \alpha v_{0}}-\frac{L^{*}}{2 \alpha v_{0}} \log \left[1-\frac{2 \alpha}{L^{*}}\left(d-\frac{\tilde{L}-L^{*}}{2 \alpha}\right)\right], & L^{*}<\tilde{L} \leqslant L^{*}+2 \alpha d, \\ d / v_{0}, & \tilde{L} \geqslant L^{*}+2 \alpha d .\end{cases}
$$

\section{APPENDIX C: AVERAGE SEGMENT LENGTH IN AN OBSTACLE FIELD}

Consider obstacles uniformly randomly distributed across a space discretized into voxels of volume $\delta V$, with an average density $\rho$. The probability of finding an obstacle in a given voxel is simply $\rho \delta V$; the probability of finding no obstacles in $N$ voxels and then one obstacle in a given further voxel reads

$$
\rho \delta V(1-\rho \delta V)^{N}
$$

Let the $N$ voxels fill a closed surface of volume $W=N \delta V$. Taking the continuum limit $\delta V \rightarrow \mathrm{d} V$ at constant $W$, we find

$$
\rho \delta V(1-\rho \delta V)^{N} \rightarrow \rho \mathrm{d} V \exp (-\rho W),
$$

We now let the volume be a right trapezoid of thickness $s$, with a fixed $\operatorname{angle} \arctan \left(h_{k} / w_{k}\right)$, maximum height $n_{\mathrm{th}} h_{k}$, and width $L$, giving a volume function (easily found geometrically) of

$$
W(L)= \begin{cases}s L^{2} h_{k} / w_{k}, & L<n_{\mathrm{th}} w_{k}, \\ s n_{\mathrm{th}}^{2} w_{k} h_{k}+n n_{\mathrm{th}} h_{k}\left(L-n_{\mathrm{th}} w_{k}\right), & L \geqslant n_{\mathrm{th}} w_{k} .\end{cases}
$$

The probability of finding such a volume of width $L$ empty and then exactly one obstacle when extending by $d L$ is given by

$$
P[\text { obs. } \in(L, L+d L)]=\rho[d W(L) / d L] \exp [-\rho W(L)],
$$

which clearly integrates to one, as eventually at least one obstacle will be found. The average width $\langle L\rangle$ is thus given by

$$
\langle L\rangle=\rho \int_{0}^{\infty} \rho[d W(L) / d L] \exp [-\rho W(L)] d L,
$$

whose evaluation is in the main text.

[1] J. P. Hirth and J. Lothe, Theory Of Dislocations (Krieger, Malabar, FL, 1991).

[2] N. Mott, Imperfections in nearly perfect crystals (Wiley, New York, 1952), p. 173.

[3] J. Friedel, Les Dislocations (Gauthier-Villars, Paris, 1956).

[4] A. Foreman and M. Makin, Philos. Mag. 14, 911 (1966).

[5] T. Nogaret and D. Rodney, Phys. Rev. B 74, 134110 (2006).

[6] S. Patinet and L. Proville, Phys. Rev. B 78, 104109 (2008).

[7] Y. Dong, T. Nogaret, and W. Curtin, Metall. Mater. Trans. A 41, 1954 (2010).

[8] A. S. Argon, Strengthening Mechanisms in Crystal Plasticity, Oxford Series on Materials Modelling (Oxford University, Oxford, 2008).

[9] N. M. Ghoniem, S. H. Tong, J. Huang, B. N. Singh, and M. Wen, J. Nucl. Mater. 307, 843 (2002).

[10] D. J. Bacon, Y. Osetsky, and D. Rodney, in Dislocations in Solids, Vol. 15, edited by J. P. Hirth and L. Kubin (Elsevier, Amsterdam, 2009), pp. 1-90.

[11] D. Terentyev, S. Hafez Haghighat, and R. Schäublin, J. Appl. Phys. 107, 061806 (2010).

[12] E. Clouet, S. Garruchet, H. Nguyen, M. Perez, and C. S. Becquart, Acta Mater. 56, 3450 (2008).

[13] L. Dupuy and M. C. Fivel, Acta Mater. 50, 4873 (2002).

[14] S. Brazovskii and T. Nattermann, Adv. Phys. 53, 177 (2004).
[15] A. Rosso, P. Le Doussal, and K. J. Wiese, Phys. Rev. B 75, 220201 (2007).

[16] S. J. Zinkle and J. T. Busby, Mater. Today (Oxford, U.K.) 12, 12 (2009).

[17] E. Gaganidze, H.-C. Schneider, B. Dafferner, and J. Aktaa, J. Nucl. Mater. 355, 83 (2006).

[18] I. Cook, Nat. Mater. 5, 77 (2006).

[19] P. B. Hirsch, S. G. Roberts, and J. Samuels, Proc. R. Soc. London, Ser. A 421, 25 (1989).

[20] P. Gumbsch, Science 282, 1293 (1998).

[21] N. Baluc, J. Boutard, S. Dudarev, M. Rieth, J. B. Correia, B. Fournier, J. Henry, F. Legendre, T. Leguey, M. Lewandowska, R. Lindau, E. Marquis, A. M. Muñoz, B. Radiguet, and Z. Oksiuta, J. Nucl. Mater. 417, 149 (2011), proceedings of ICFRM-14 .

[22] V. Vitek, Phys. Status Solidi 18, 687 (1966).

[23] A. Seeger and P. Schiller, in Physical Acoustics, Principles and Methods, edited by W. P. Mason (Academic, New York, 1966), pp. 361-495.

[24] S. Queyreau, J. Marian, M. R. Gilbert, and B. D. Wirth, Phys. Rev. B 84, 064106 (2011).

[25] L. Proville, D. Rodney, and M. C. Marinica, Nat. Mater. 11, 845 (2012).

[26] D. Cereceda, M. Diehl, F. Roters, D. Raabe, J. M. Perlado, and J. Marian, Int. J. Plast. 78, 242 (2016). 
[27] G. Po, Y. Cui, D. Rivera, D. Cereceda, T. D. Swinburne, J. Marian, and N. Ghoniem, Acta Mater. 119, 123 (2016).

[28] D. Brunner, Mater. Trans., JIM 41, 152 (2000).

[29] M. R. Gilbert, P. Schuck, B. Sadigh, and J. Marian, Phys. Rev. Lett. 111, 095502 (2013).

[30] G. Monnet, S. Naamane, and B. Devincre, Acta Mater. 59, 451 (2011).

[31] F. Louchet, L. Kubin, and D. Vesely, Philos. Mag. A 39, 433 (1979).

[32] M. Charleux, F. Livet, F. Bley, F. Louchet, and Y. Brechet, Philos. Mag. A 73, 883 (1996).

[33] O. M. Braun and Y. S. Kivshar, The Frenkel-Kontorova Model: Concepts, Methods, and Applications, Texts and Monographs in Physics (Springer, Berlin, 2004).

[34] K. G. Fedorov and A. L. Pankratov, Phys. Rev. Lett. 103, 260601 (2009).

[35] E. Orowan, Proc. Phys. Soc. 52, 8 (1940).

[36] T. D. Swinburne, S. L. Dudarev, S. P. Fitzgerald, M. R. Gilbert, and A. P. Sutton, Phys. Rev. B 87, 064108 (2013).

[37] A. A Giannattasio, M. Tanaka, T. D. Joseph, and S. G. Roberts, Phys. Scr., T 128, 87 (2007).
[38] L. Dezerald, L. Proville, L. Ventelon, F. Willaime, and D. Rodney, Phys. Rev. B 91, 094105 (2015).

[39] T. D. Swinburne, Phys. Rev. E 88, 012135 (2013).

[40] P. Hänggi, P. Talkner, and M. Borkovec, Rev. Mod. Phys. 62, 251 (1990).

[41] L. Dezerald, L. Ventelon, E. Clouet, C. Denoual, D. Rodney, and F. Willaime, Phys. Rev. B 89, 024104 (2014).

[42] M. C. Marinica, L. Ventelon, M. R. Gilbert, L. Proville, S. L. Dudarev, J. Marian, G. Bencteux, and F. Willaime, J. Phys.: Condens. Matter 25, 395502 (2013).

[43] T. D. Swinburne and M. C. Marinica, Phys. Rev. Lett. 120, 135503 (2018).

[44] X. Yi, A. E. Sand, D. R. Mason, M. A. Kirk, S. G. Roberts, K. Nordlund, and S. L. Dudarev, Europhys. Lett. 110, 36001 (2015).

[45] T. D. Swinburne, P.-W. Ma, and S. L. Dudarev, New J. Phys. 19, 073024 (2017).

[46] T. D. Swinburne, K. Arakawa, H. Mori, H. Yasuda, M. Isshiki, K. Mimura, M. Uchikoshi, and S. L. Dudarev, Sci. Rep. 6, 30596 (2016). 